\title{
Distinguishing dynamic and static yield stress of fresh cement mortars through thixotropy
}

\author{
Ye Qian, Shiho Kawashima* \\ Columbia University, Department of Civil Engineering and Engineering Mechanics, 500 West 120th Street, New York, NY 10027, USA
}

\section{A R T I C L E I N F O}

\section{Article history:}

Received 14 December 2016

Received in revised form

31 July 2017

Accepted 27 November 2017

Available online 2 December 2017

\section{Keywords:}

Dynamic yield stress

Static yield stress

Thixotropy

Negative slope

Shear banding

Mortar

\begin{abstract}
A B S T R A C T
The dynamic and static yield stress of fresh cement mortar were measured in a rotational rheometer with a vane geometry using shear rate and shear stress-controlled protocols, respectively. Through a shear rate-controlled steady-state protocol, the equilibrium flow curve is measured and fitted with the Bingham model to obtain dynamic yield stress. A negative slope in the equilibrium flow curve, shear banding and stick-slip phenomena are observed and discussed. Through a stress-controlled creep-recovery protocol, viscosity bifurcation behavior is captured and static yield stress is marked as the creep stress when the bifurcation occurs. Finally, the discrepancy between dynamic and static yield stress is tied to thixotropy.
\end{abstract}

๑) 2017 Published by Elsevier Ltd.

\section{Introduction}

Yield stress has been extensively studied in non-Newtonian fluids, including colloidal gel, bentonite, polymer suspension, food, paints, waxy crude oil, pastes, and foams [1-5]. Specifically in cementitious materials, yield stress is important in quantifying flowability [6] and is correlated to conventional field-friendly measurement methods such as the slump and slump flow test, both experimentally and in simulation [7-10].

As a common method to obtain yield stress, an equilibrium flow curve is plotted as the equilibrium shear stress - shear rate relationship. Since the publication of the Bingham model [11], many models have been proposed to describe the equilibrium flow curve. One of the most common models for cementitious materials is the Bingham model [12-14], $\sigma=\sigma_{y}+\eta_{p l} \dot{\gamma}$, where $\sigma$ is the shear stress, $\dot{\gamma}$ is the shear rate, $\sigma_{y}$ is the Bingham yield stress and $\eta_{p l}$ is the plastic viscosity. As a linear model, it is unable to capture shear thinning and shear thickening behaviors that can be observed in cementitious materials. Thus, the Herschel-Bulkley model is also commonly used, $\sigma=\sigma_{y}+k \dot{\gamma}^{n}$, where $\mathrm{k}$ and $\mathrm{n}$ are constants

\footnotetext{
* Corresponding author. Columbia University, Department of Civil Engineering and Engineering Mechanics, 500 West 120th street, 616 MUDD, New York, NY 10027, USA.

E-mail address: s-kawashima@columbia.edu (S. Kawashima).
}

describing material properties, and $\mathrm{n}<1$ for shear thinning and $\mathrm{n}>1$ for shear thickening [15,16]. Studies by Yahia and Khayat [17] show that a modified Bingham model, $\sigma=\sigma_{y}+\eta_{p l} \dot{\gamma}+c \dot{\gamma}^{2}$, also provides a good description of non-linear flow behavior. With each of these models, a measure of yield stress can be obtained.

The yield stress of cement paste originates from the microstructure of particle-particle network through colloidal interaction or direct contact. The microstructure sustains a certain amount of stress before it is broken down and starts to flow, which defines the yield stress. At rest, the microstructure builds up due to colloidal flocculation and cement hydration bonding, such as CSH bridges [18], thus resulting in higher yield stress. Under shear flow, the structure and bonding network is broken down, resulting in lower yield stress. This property of fresh cementitious materials, i.e. before setting, where the microstructure and yield stress changes with flow history is termed thixotropy [19-22]. Roussel [18] assumed that the hydration products, even at early ages, contributes to the structuration of fresh cement paste. Early hydration products on the surface of cement particles bridge these particles together to form a rigid network, which exhibits a relatively small critical strain. In comparison, colloidal forces of cement powders contribute to a softer network with a relatively higher critical strain. These two networks both act as the origin of thixotropy of cement paste. They also play a big role in structuration and yield stress. Thus, yield stress and thixotropy are closely related $[23,24]$. 
It could be reasoned that because of thixotropy there should be more than one state of flocculation or microstructure when yield stress is measured, depending on the flow history of the material. Since generally the equilibrium shear stress increases with shear rate, the yield stress measured through equilibrium flow curve is regarded as the shear stress when shear rate is 0 , so the minimum stress to sustain or terminate the flow of the material. This is commonly termed dynamic yield stress, and so is the yield stress that is obtained through the aforementioned steady-state flow models. Meanwhile, there exists a yield stress corresponding to a state before the structure is broken down, so the stress necessary to initiate flow, corresponding to a well-connected undisturbed microstructure $[23,25]$. This is considered to be the static yield stress. One method to measure the static yield stress is to apply creep-recovery, which was implemented by Struble and Schultz [15] and later by the authors [26]. This is a stress-controlled test where the material will not flow until the applied creep stress is higher than its static yield stress. The static yield stress is expected to be higher than dynamic yield stress considering the corresponding structural states. The existence of separate dynamic and static yield stresses in the same material is known in cement-based systems, as well as bentonite [27], paints [28] and waxy crude and fuel oils [29].

Dynamic yield stress is commonly measured and studied because the equilibrium flow curve is a convenient, wellestablished method of shear rheological characterization. Measurements taken in field tests [30], such as the slump flow test, are also tied to dynamic yield stress [7,8,31]. Meanwhile, static yield stress is also very important and critical for concrete rheology and SCC studies. For example, formwork pressure [13,32-37], stability [38], distinct layers casting [39] of SCC are closed related to structural rebuilding, which is directly related to static yield stress. Static yield stress is also of great interest for 3D concrete printing applications $[39,40]$.

In this study, flow behavior of mortar under constant shear rate and constant stress is studied. Mortar systems are selected as they exhibit both viscous and granular behavior, similar to concrete, but can be probed with high precision rotational rheometers. Through constant applied shear rate, the equilibrium flow curve and dynamic yield stress are obtained. Under constant applied stress, performed in the creep-recovery protocol, static yield stress is measured. The discrepancy between static and dynamic yield stress is related to thixotropy and it is explicitly demonstrated and discussed that the dependence of measured yield stress on preshear condition is due to thixotropy.

\section{Material and procedures}

The materials, rheometer, and mixing and testing protocols are described in detail in another study [41] and only briefly explained here.

All mortars are prepared with Type I Portland cement, ovendried sand between 0.6 and $1.18 \mathrm{~mm}$ in diameter, highly purified attapulgite clay, i.e. nanoclay, and water are used. No chemical admixtures are used. The water-to-cement ratio $(\mathrm{W} / \mathrm{C})$ is 0.5 by mass. The sand-to-cement ratio $(\mathrm{S} / \mathrm{C})$ is set to $2: 1$ by mass, yielding a sand volume fraction of approximately $48 \%$. The sand is river sand, sieved through ASTM \#30 and \#50 sieves, thus the diameter is between 0.6 and $1.18 \mathrm{~mm}$. The water absorption rate is $1.55 \%$, which is considered when proportioning to achieve the desired $\mathrm{w} / \mathrm{c}$ ratio. Nanoclay was incorporated into the mixes because previous studies by the authors showed that it alleviates sand migration and enhances static stability of fresh mortars [41]. Nanoclay is added at $0.5 \%$ by mass of cement, which is found to be sufficient in achieving statically stable mixes with no visible signs of bleeding.
Nanoclay powder is first blended with water for 2 min to produce a suspension. Cement powder is slowly poured into the solution and mixed at a speed of $136 \mathrm{rpm}$ for $1 \mathrm{~min}$, then at a higher speed of $281 \mathrm{rpm}$ for an additional $4 \mathrm{~min}$. Then, sand is slowly poured into the cement paste and hand-mixed in a random manner for $4 \mathrm{~min}$. Immediately after mixing, the fresh mortar is poured into the construction cell. Before each test, the mortar is strongly tampered by hand mixing in a random manner for 1 min to bring the material to a destructed, homogeneous state. More detailed discussion on the hand-mixing approach are presented in Refs. $[25,41]$.

The rheometer is a HAAKE MARS III rheometer. The construction cell is a cylinder with the diameter of $74 \mathrm{~mm}$ and the height of $150 \mathrm{~mm}$, with 24 profiles of $2 \mathrm{~mm}$ evenly distributed along the wall to prevent wall slip. The rotor is a two blade vane with a diameter of $52 \mathrm{~mm}$ and height of $50 \mathrm{~mm}$. More details are provided in Ref. [41].

In this study, torque ( $\mathrm{mNm})$, angular velocity ( $\mathrm{rad} / \mathrm{s})$ and angular deformation (rad) are used. Other studies have focused on processing raw data, such as torque and angular velocity to shear stress and shear rate, respectively. The Reiner-Riwlin equation is commonly used for this transformation [42], which assumes Bingham behavior for flow. Because of the non-linear flow behavior of cementitious materials, Feys et al. [43] extended the ReinerRiwlin equation and used a modified Bingham model to transform raw coaxial cylinder data. Other methods such as Estelle et al. [44] also use a linear model to process the transformation. However, due to the complexities of the flows observed (i.e. shear banding, stick slip, shear localization), results are presented in torque, angular velocity, and angular deformation. This is believed to be sufficient for discussion of the presented work.

In all tests, at least three samples were tested and the average was taken to be the representative value.

\section{Measuring dynamic yield stress through shear rate- controlled protocol}

\subsection{Equilibrium flow curve: using constant angular velocity protocol}

Here, dynamic yield stress is obtained by fitting the equilibrium flow curve with a steady-state flow model. For each step, the fresh mortar is subjected to $1 \mathrm{~min}$ of strong hand mixing, then a constant angular velocity is applied for $60 \mathrm{~s}$ during which the corresponding torque decay is recorded. For each run, the torque development with time is as follows: the torque increases from 0 to a peak value and then decreases to an equilibrium value over time, as shown in Fig. 1. Constant angular velocity is applied between 1 and $50 \mathrm{rad} / \mathrm{s}-$ it is verified that $60 \mathrm{~s}$ is sufficient to reach torque equilibrium within this range. It is observed that the material response differs between higher velocities, i.e. 7-50 rad/s, and intermediate velocities, i.e. $1-7 \mathrm{rad} / \mathrm{s}$.

From 7 to $50 \mathrm{rad} / \mathrm{s}$, the equilibrium torque increases with increasing angular velocity. The resultant equilibrium flow curve can be obtained by plotting the equilibrium torque and corresponding applied angular velocity, and is presented in Fig. 2. It is depicted that from $7 \mathrm{rad} / \mathrm{s}$ to $50 \mathrm{rad} / \mathrm{s}$ the flow curve can be fitted with the Bingham model:

$T=0.5033 \cdot \Omega+41.72$

where $T$ is torque in $\mathrm{mNm}$ and $\Omega$ is angular velocity in $\mathrm{rad} / \mathrm{s}$. According to the fitting, with a $\mathrm{R}^{2}$ of $0.9741,41.72 \mathrm{mNm}$ is the dynamic yield stress.

It must be noted that shear-induced particle migration is a critical issue in fresh mortar systems under flow and has long been 


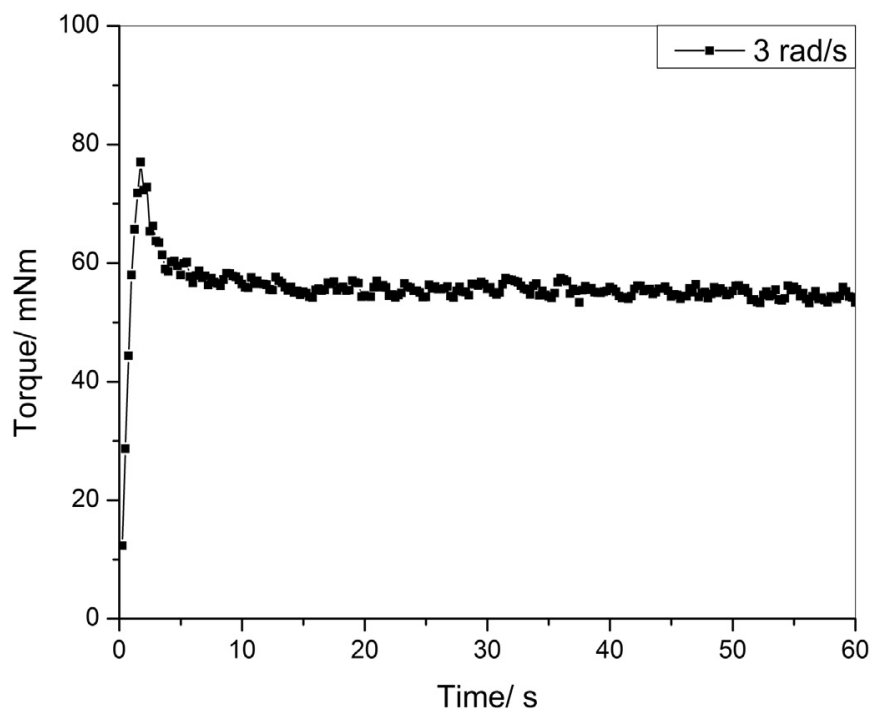

Fig. 1. Stress development under constant shear rate.

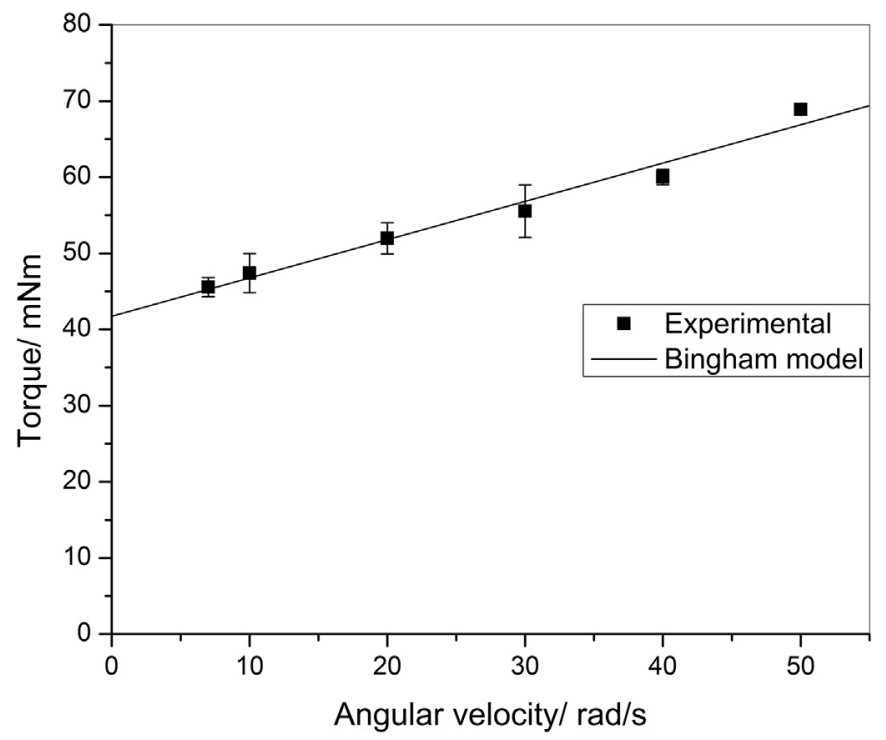

Fig. 2. Equilibrium flow curve of mortar.

observed and discussed [45-48]. Under shear, sand particles in the paste matrix migrate from regions of high shear rate to low shear rate. In this study, this translates to sand particles migrating from the sheared region to the unsheared region, i.e. from the vane rotor to the wall. Studies by Assaad et al. $[13,49]$ showed that slotted devices can help to mitigate migration of large particles. However, in a separate study testing the same mortar system, the authors still observed particle migration and proposed an empirical model to quantify how its kinetics and magnitude are affected by various parameters [41]. Based on those findings, here it is expected that sand migration is occurring. So although the material reaches an equilibrium state at each applied constant angular velocity and the steady-state flow curve can be obtained, the dynamic yield stress is likely an underestimation of the fresh mortar system had it remained uniform. More work needs to be done to obtain representative shear rheological measurements and to mitigate dynamic segregation of mortars.

In contrast, between 1 and $7 \mathrm{rad} / \mathrm{s}$, the equilibrium torque

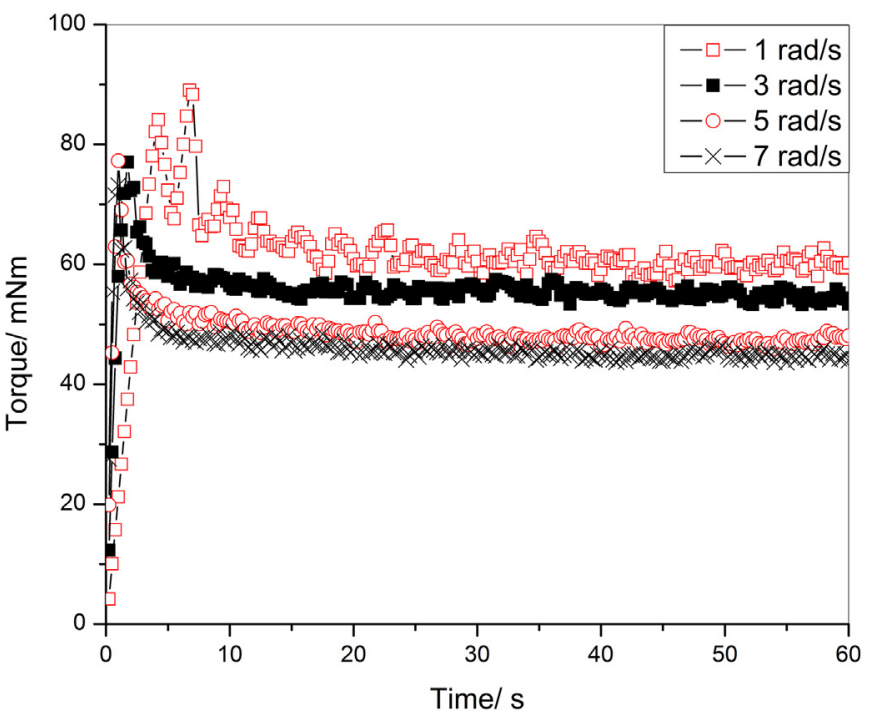

Fig. 3. Torque decay at constant angular velocity between $1 \mathrm{rad} / \mathrm{s}$ and $7 \mathrm{rad} / \mathrm{s}$.

decreases with increasing applied angular velocity, as shown in Fig. 3. Combining the flow curve presented in Fig. 2, the full equilibrium flow curve of the fresh mortar is shown in Fig. 4. Different from most literature, we observe a negative slope in the equilibrium flow curve. Moller et al. [50] found this negative slope in soft glassy materials, such as foams, emulsions, and colloidal gels. To the knowledge of the authors, a discussion or experimental results on a negative slope in the equilibrium flow curve of cement-based systems has not been published. Further, Moller et al. [50] did not discuss the mechanisms underlying why shear stress is smaller at higher applied shear rates.

It has been discussed and verified that for yield stress fluids (e.g. clay suspension and carbopol gel), shear localization occurs [50-52]. When the applied shear rate is below a critical value, only part of the material in the vicinity of the surface of the rotor is sheared while the rest remains unsheared. There is a critical radius, $r_{c}$, distinguishing these two regions. Through magnetic resonance imaging (MRI), Jarny, Roussel et al. [52]. verified these phenomena in white cement pastes. They measured the tangential speed of cement paste in a rheometer with a couette geometry, as depicted in Fig. 5. In steady state, the tangential speed of cement paste linearly decreases from the surface of the inner rotor and reaches zero at $r_{c}$. Since the differential of tangential speed over the radius is linear, the effective shear rate is uniform throughout the entire sheared region. Furthermore, at various angular velocities the tangential speed profiles exhibit the same slope, which means that the effective shear rate remains unchanged. The difference is that with increasing angular velocity of the rotor $r_{c}$ increases, corresponding to larger sheared region.

It is reasoned that for the fresh mortars tested in this study, at low shear rates, i.e. 1-7 rad/s, shear localization occurs: only part of the material in the vicinity of the rotor is sheared and the rest remains unsheared. The increase in the apparent global shear rate does not increase the effective shear rate of the sheared region - it only increases the critical radius. As aforementioned, shear-induced sand migration is expected to be occurring, even within this intermediate angular velocity range. So with increasing angular velocity increasingly more material is sheared and, subsequently, more sand migrates to the unsheared region, effectively lowering the density and viscosity of the sheared region. Considering that the effective shear rate at various applied angular velocities is the same, the decrease in viscosity of the sheared region leads to a 


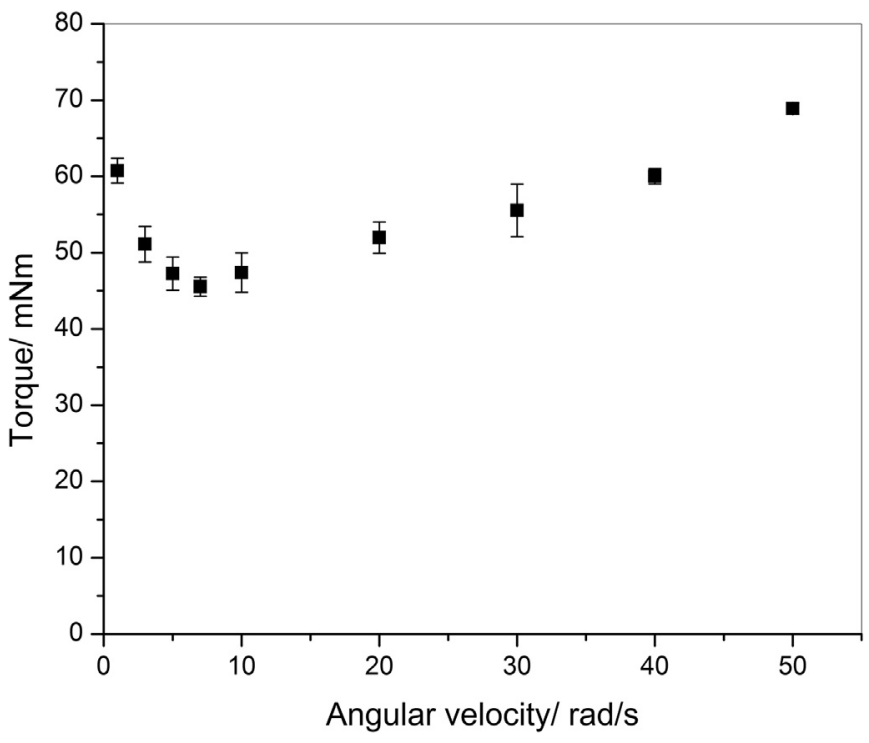

Fig. 4. Complete equilibrium flow curve of mortar.

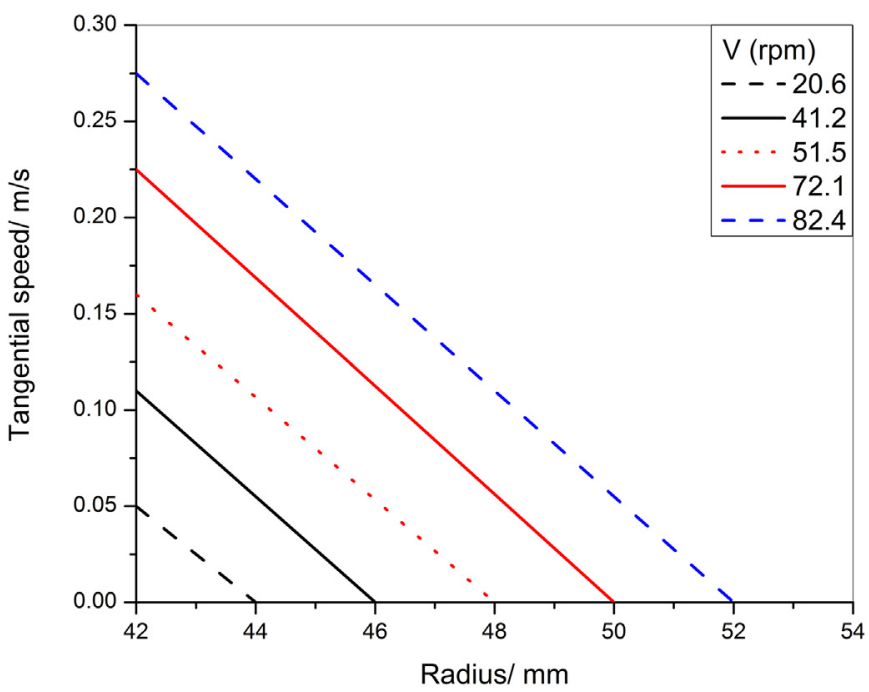

Fig. 5. Tangential velocity diagram under constant apparent shear rate, adopted from Jarmy, Roussel et al. [40].

decrease in torque. Thus shear localization and shear-induced sand migration at least partially explains the negative slope observed in the steady-state flow curve of fresh mortars.

\subsection{Stick slip: flow behavior under low shear rate}

At low applied angular velocity, $0.1 \mathrm{rad} / \mathrm{s}$, the torque does not develop continuously with shearing time, as shown in Fig. 6(a). The torque increases linearly with time and then suddenly drops incrementally. Concurrently, the rotational velocity of the rotor also jumps from near 0 to a finite value and then back to 0 again. Additionally, it can be seen from Fig. 6(b) that the angular deformation of the rotor does not increase linearly with time. The angular deformation is step-wise, where it increases incrementally then remains nearly constant. These observations indicate that stick slip, which is abrupt flow and stop, is occurring.

This stick slip behavior could be related to the phenomenon theoretically described as shear banding by Coussot et al. [53]. At
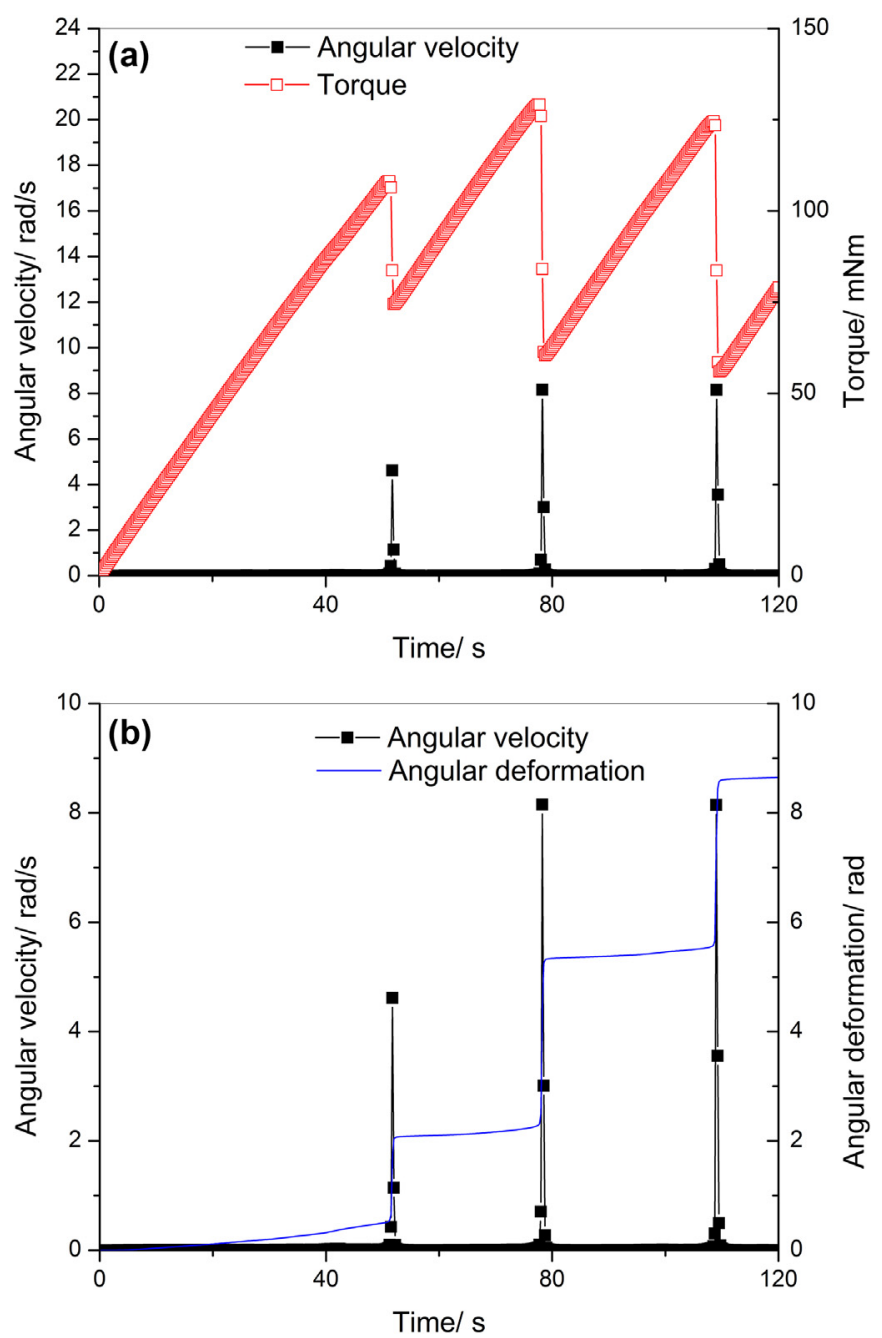

Fig. 6. Under applied angular velocity $0.1 \mathrm{rad} / \mathrm{s}$, (a) angular velocity and torque development; (b) angular velocity and angular deformation.

the beginning of constant shear rate and shearing process, the material is solid-like and fractures in the local "weak" regions where the critical stress is exceeded. During fracture, the alignment of material, especially granular particles in mortar, is adjusted. So the sudden jump of angular velocity and torque is recorded. With further shearing, stick slip occurs repeatedly because there are many local "weak" regions fracturing one after another. So compared with the material before shearing, there are many fracture bands forming in the material.

If deformation due to stick slip is disregarded, the angular deformation throughout the shearing period is very low. The slight increase in angular deformation before the first stick slip occurs is due to the viscoelastic deformation of the material. The angular deformation before the first stick slip occurs can be considered as the critical fracturing angular deformation, beyond which the microstructure is broken and fracture occurs. It is averaged at $0.697 \mathrm{rad}$ for the mortar system tested in this study, and will be tied to static yield stress as measured by creep recovery protocol in Section 4.2 .

\subsection{Theoretical discussion of material response under constant shear rate}

To sum up, as proposed by Coussot et al. [53] and further 


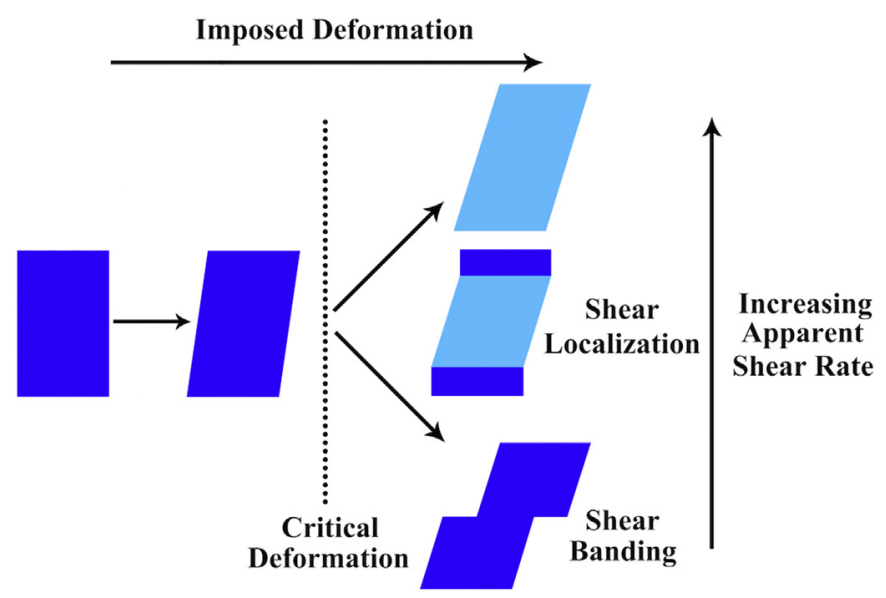

Solid Region

Flowing Region

Fig. 7. Schematic of deformation under increasing apparent shear rate.

confirmed by experiment results in this study, the stages of deformation and corresponding viscoelastic regimes will be discussed. A schematic of deformation under increasing apparent shear rate is shown in Fig. 7. When subjected to shear, the material initially deforms as a viscoelastic jamming solid, exhibiting only a finite shear deformation. Then, beyond a critical deformation, shear behavior will differ depending on the magnitude of applied angular velocity. At very low applied angular velocities, shear banding and stick slip occur. At intermediate velocities, shear localization occurs where part of the material near the rotor is sheared at a critical effective shear rate while the rest remains unsheared. Within this range, the critical radius increases with increasing angular velocity. Finally, at sufficiently high velocities, the entire material is sheared and the higher the applied angular velocity, the higher the effective shear rate and torque. In other words, in this shear rate regime, shear stress becomes a function of shear rate fitted with a Bingham model.

\section{Measuring static yield stress through shear stress- controlled protocols}

\subsection{Stress growth protocol}

The stress growth protocol is commonly implemented to measure static yield stress, where a linearly increasing shear stress is applied and the shear strain is recorded. Once the structure is broken and solid-liquid transition occurs, the strain dramatically increases. Thus the corresponding applied stress is the static yield stress. The protocol applied is shown in Fig. 8(a). Three increasing rates of stress are applied: $20,6.7$ and $3.3 \mathrm{mNm} / \mathrm{s}$. The results in Fig. 8(b) show that the static yield stress values measured are 85 , 110 and $140 \mathrm{mNm}$, respectively. It is apparent that the yield stress measured by the linearly increasing stress protocol is highly dependent on the increasing rate. The lower the loading rate, the higher the measured yield stress. This can be attributed to thixotropy and hydration: at lower loading rate, the material has more time to rebuild its structure so the yield stress to break the structure is higher.

\subsection{Static yield stress: $u$ sing creep recovery protocol}

To eliminate the rate-dependence of the stress growth protocol, we implement a creep-recovery protocol to measure static yield
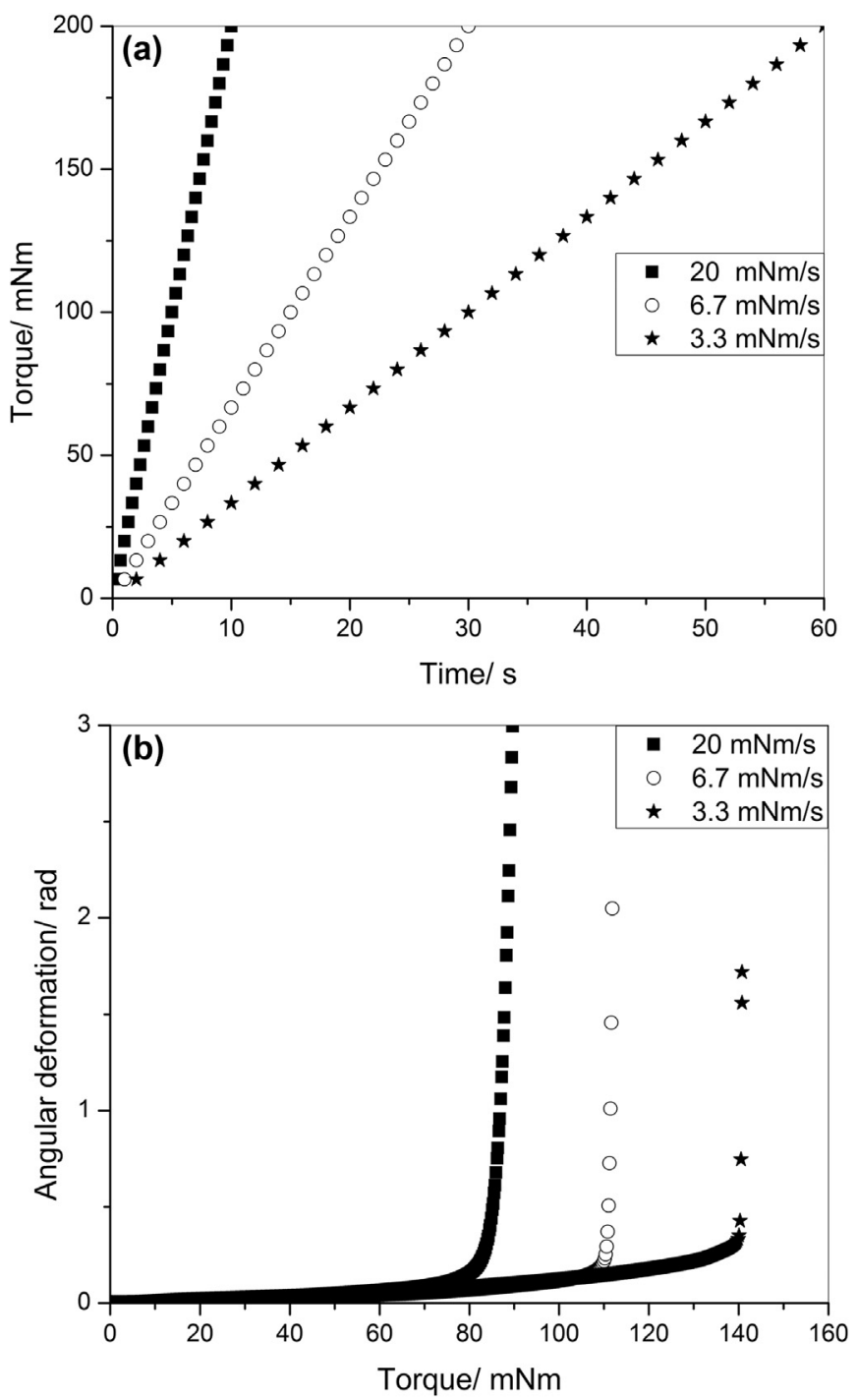

Fig. 8. (a) Linearly increasing stress protocol and (b) corresponding angular deformation vs torque results.

stress, which was applied to paste systems in other work by the authors [26]. Constant shear stress is applied and held for a period of time, then completely removed. The resulting strain and strain rate evolution are recorded. Within a certain range of applied torques, angular deformation eventually ceases during the creep step and the material exhibits some degree of recovery, as shown in Fig. 9. On the other hand, beyond a critical applied torque, the material undergoes continuous deformation during creep and exhibits no recovery, as shown in Fig. 10.

First, the creep portion of the protocol will be discussed. Comparing (a) and (b) of Fig. 9, it is apparent that the angular deformation reaches equilibrium at higher values when under higher creep torque. Fig. 11 presents the equilibrium angular deformation measured during the creep step at different applied torques, capturing the linear and nonlinear regimes of creep deformation. When torque is low (below $50 \mathrm{mNm}$ in this study), the creep behavior is linear. As can be seen in the inset of Fig. 11(a), angular deformation is proportional to torque, which means the creep compliance (the ratio of strain over stress, here angular deformation over torque) is constant. With increasing torque the angular deformation increases dramatically and reaches 

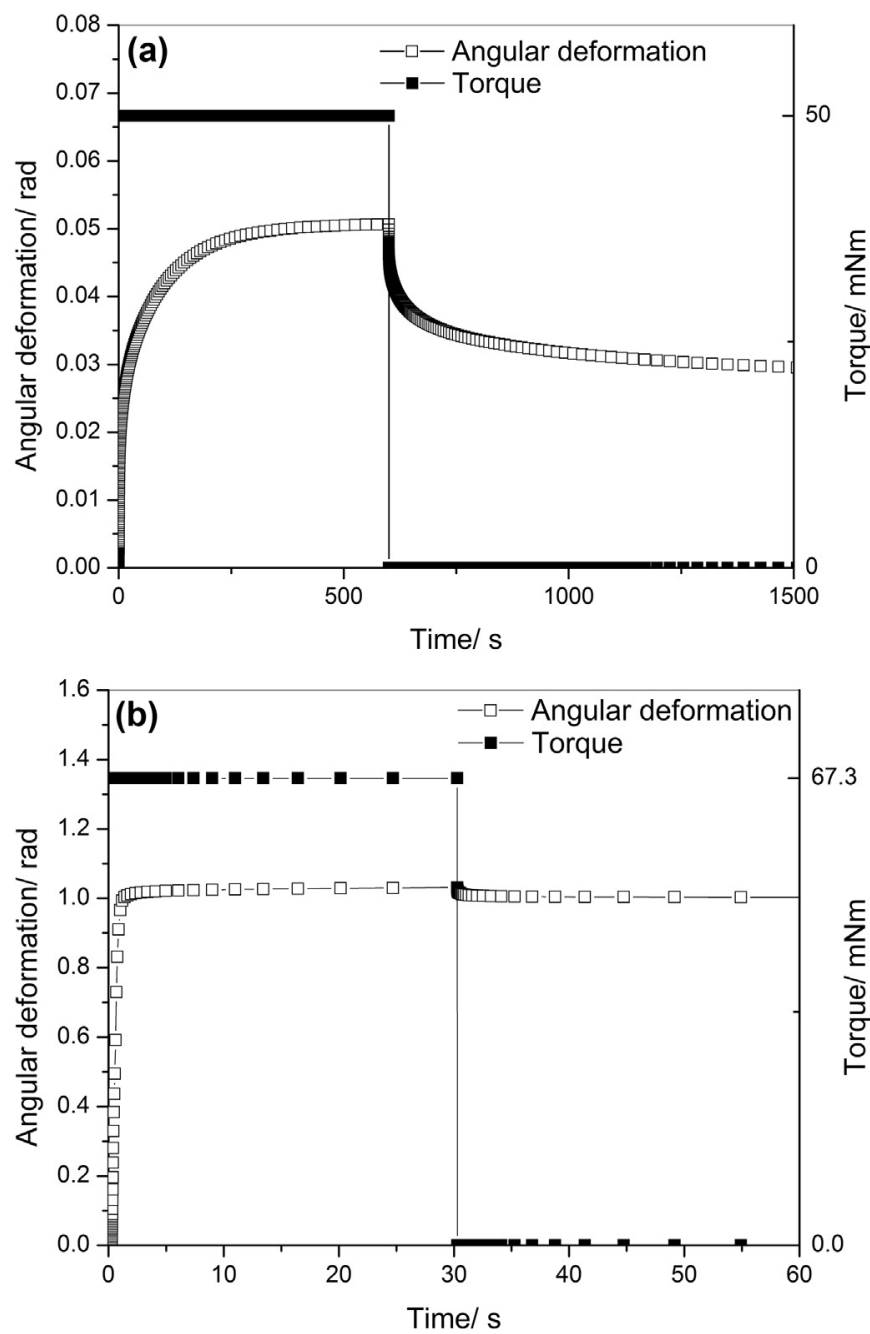

Fig. 9. Creep recovery behavior of mortar under torque at (a) $50 \mathrm{mNm}$; (b) $67.3 \mathrm{mNm}$.

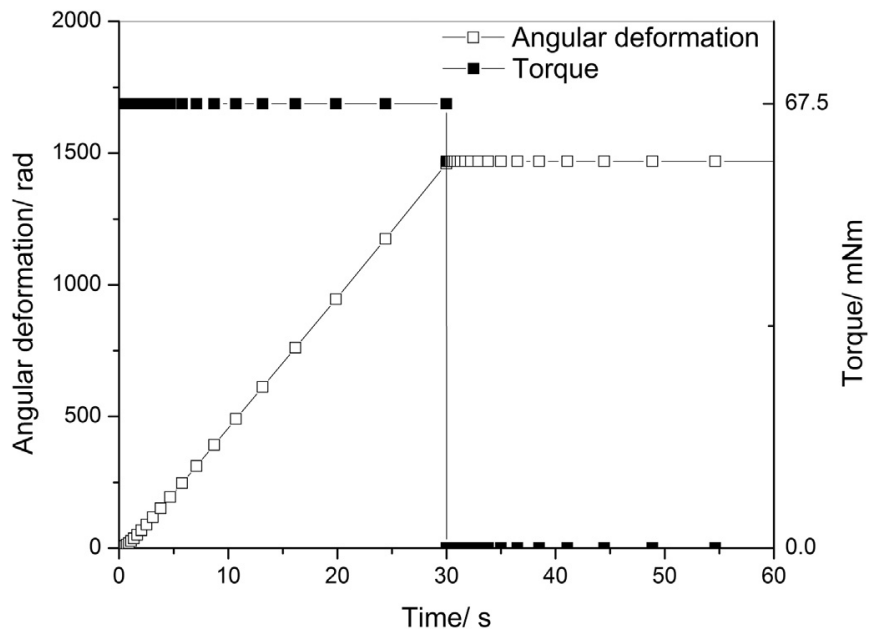

Fig. 10. Creep recovery behavior of mortar under torque at $67.5 \mathrm{mNm}$.

equilibrium more rapidly, thus the creep behavior becomes highly nonlinear, indicating yielding deformation. At a slightly higher applied torque of $67.5 \mathrm{mNm}$, the linear increase of angular deformation shows that the material is flowing, as shown in Fig. 10.
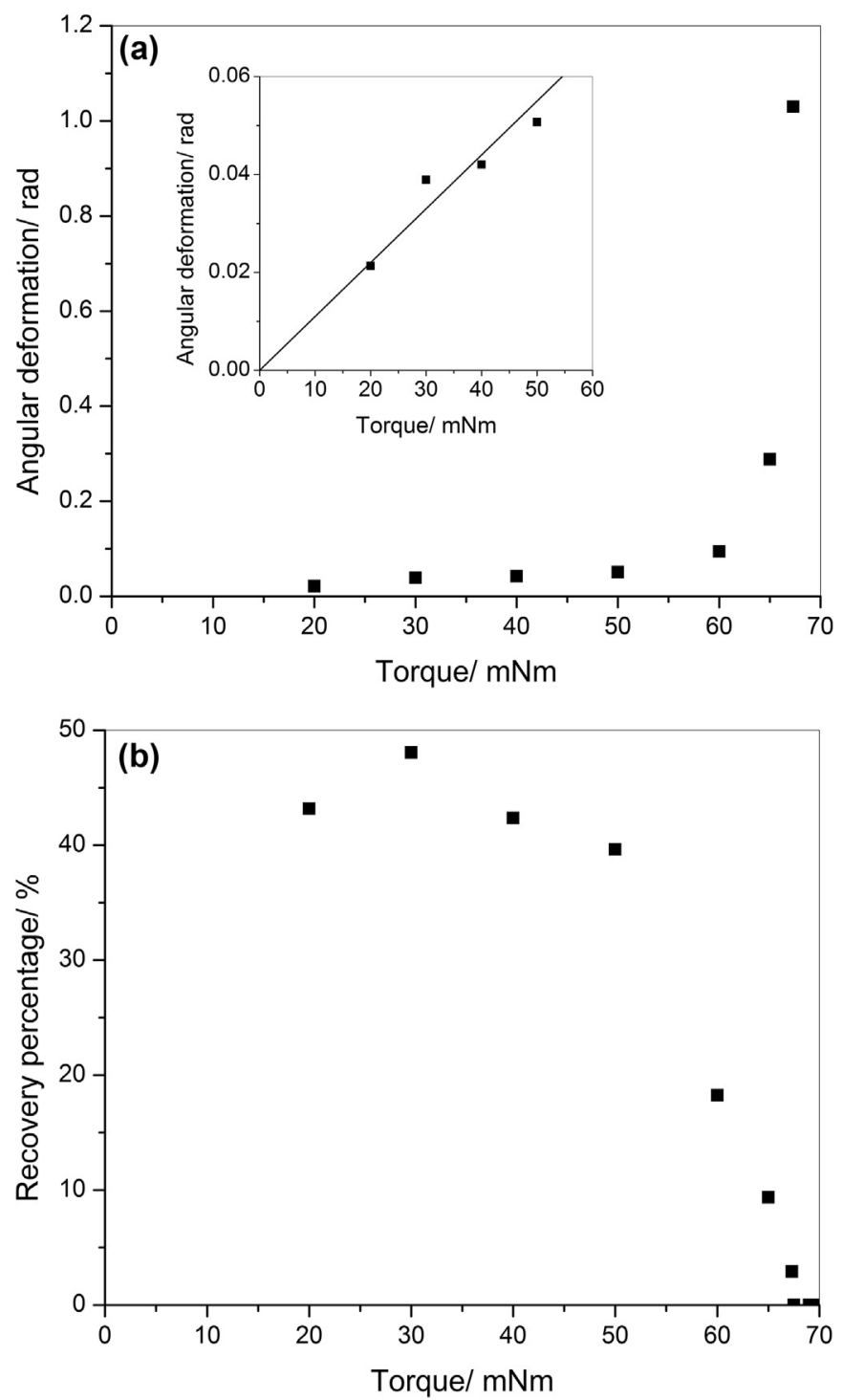

Fig. 11. (a) Angular deformation vs torque and (b) recovery percentage vs torque.

It is summarized that there are two critical torques during creep. One is the critical linear-deformation torque, below which the creep compliance is constant and independent of the applied torque $-50 \mathrm{mNm}$. The other is the critical shearing torque, beyond which the material shears at a constant angular velocity-67.5 $\mathrm{mNm}$. It is the latter, which is tied to static yield stress.

When the torque is released to 0 after the creep step, the angular deformation will decrease, indicating recovery of structure. Due to the viscoelastic and thixotropic properties of the material, the angular deformation or strain does not recover fully to $100 \%$. Here the recovery percentage is quantified as

$R P=\frac{\delta_{\text {creep }}-\delta_{\text {recovery }}}{\delta_{\text {creep }}} * 100$

where $\delta_{\text {creep }}$ and $\delta_{\text {recovery }}$ are the equilibrium angular deformation at the end of creep protocol and recovery protocol.

It could be seen in Fig. 11(b) that within the critical lineardeformation torque, $50 \mathrm{mNm}$ in this study, the recovery percentage does not change much with increasing torque. And beyond that the recovery percentage decreases with increasing torque, 
indicating higher degree of structural breakdown of the material during creep until reaching 0 at the critical shearing torque, which is $67.5 \mathrm{mNm}$ in this study. Beyond the critical shearing torque the recovery rate is $0 \%$, indicating continuous flow of the material and no recovery after torque release.

Fig. 12 shows the development of angular velocities during creep. It is apparent that bifurcation occurs, similar to the phenomena described as viscosity bifurcation, or "avalanche behavior," by Coussot et al. [54]. The authors also found this phenomenon for cement paste [26]. At torques lower than $67.3 \mathrm{mNm}$, the angular velocity vanishes to zero. In contrast, at a slightly higher torque, for instance $67.5 \mathrm{mNm}$, the angular velocity increases to a constant elevated value. This angular velocity bifurcation marks a critical shearing torque. It is noted that under an applied torque of 67.5 $\mathrm{mNm}$ the angular velocity reaches an equilibrium value averaged at $50.9 \mathrm{rad} / \mathrm{s}$. Recalling the equilibrium flow curve fitted with the Bingham model at $67.5 \mathrm{mNm}$ the angular velocity is $51.2 \mathrm{rad} / \mathrm{s}$, confirming this regime is under steady-state flow and independent of shear rate or stress control.

After a plateau in the beginning of creep, an abrupt transition of angular velocity occurs. The angular velocity decreases 3 orders of magnitude within $1 \mathrm{~s}$ at torques smaller than $67.3 \mathrm{mNm}$, indicating that the system jams. At slightly higher torque, higher than the critical shearing torque, the angular velocity increases to a constant, elevated value, indicating that the system flows. Studies by Assaad et al. [55] indicated that a slotted vane, as used in this study, helped lead to abrupt breakdown of structure. Here, the transitional angular deformation is recorded from Fig. 12, considered to be when angular velocity becomes $20 \%$ higher or lower than the initial plateau value at each creep torque. As shown in Fig. 13, the transitional angular deformations increase with increasing torque.

The critical fracturing angular deformation is a parameter showing that the structure of the material is broken down. In this study, it is measured at low shear rate $0.1 \mathrm{rad} / \mathrm{s}$ in Fig. 6(b) when the first stick slip occurs due to shear banding, which is averaged at 0.697 rad. In Fig. 13, transitional angular deformations under various torques are compared with the critical fracturing angular deformation. For creep torques lower than $67.5 \mathrm{mNm}$, the transitional angular deformation is smaller than the critical fracturing angular deformation so the structure is not broken down and flow stops. For creep torque higher than $67.5 \mathrm{mNm}$, the angular deformation is higher than the critical fracturing angular deformation so

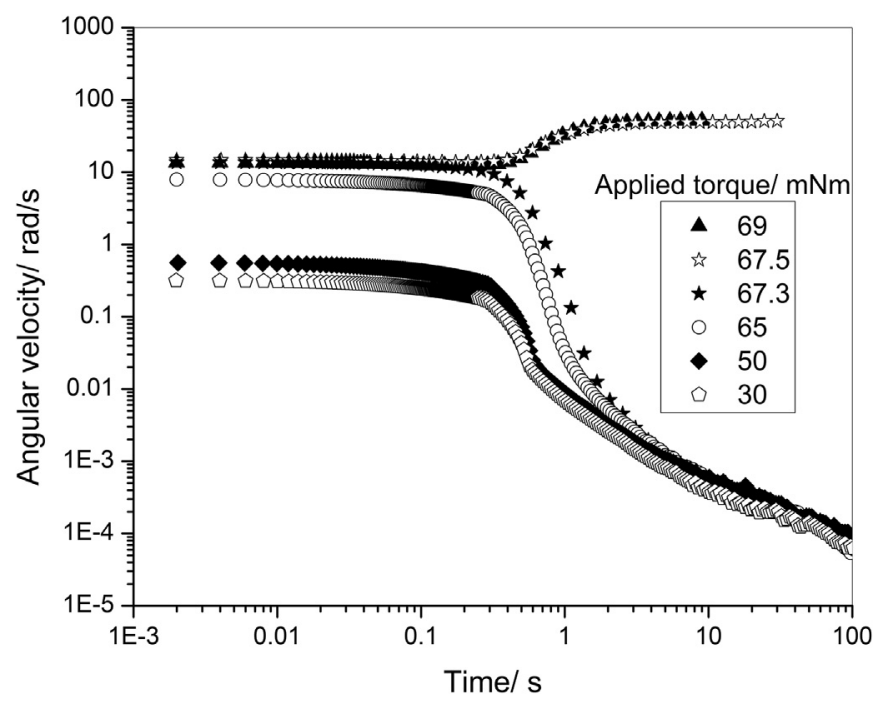

Fig. 12. Angular velocity of mortar under creep at various torque values.

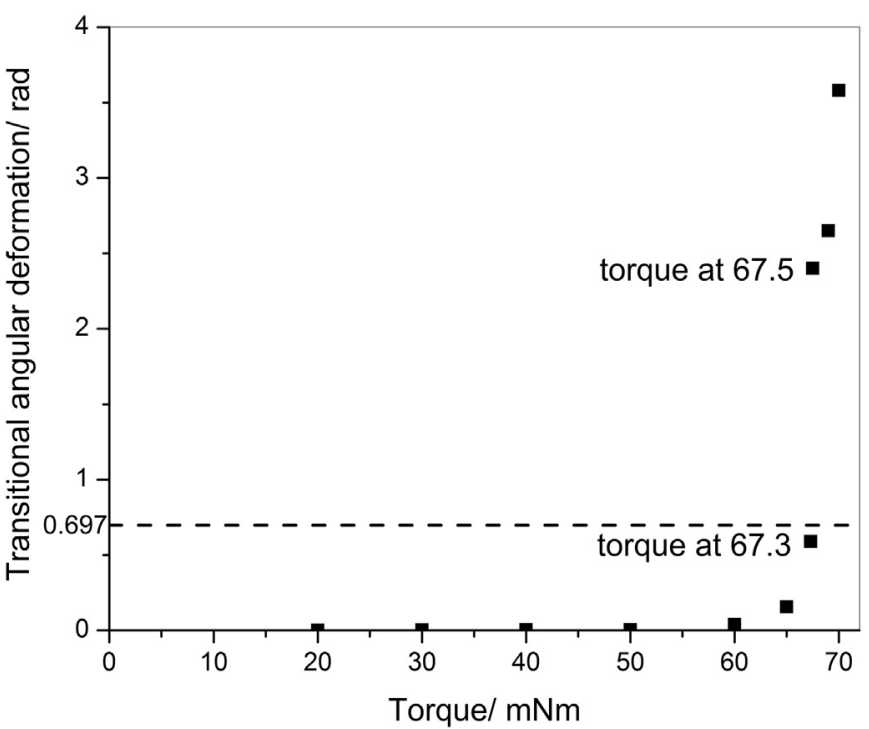

Fig. 13. Transitional angular deformation of mortar under constant torque compared to critical fracturing angular deformation.

the structure starts to break down, solid-liquid transition occurs, and the mortar continues flowing due to shear rejuvenation. Only when applied creep stress exceeds the critical stress, $67.5 \mathrm{mNm}$ in this study, can solid-liquid transition occur and the flow continue. Thus, the critical shearing torque, $67.5 \mathrm{mNm}$ in this study, is the static yield stress.

\section{Thixotropy: connecting dynamic and static yield stress}

For thixotropic systems like cement-based materials, creep behavior will depend on the initial flocculation state. In section 3.1, dynamic yield stress is obtained by fitting the equilibrium flow curve with the Bingham model- $41.72 \mathrm{mNm}$. Meanwhile, in section 4.2 static yield stress is obtained through the creep recovery protocol-67.5 $\mathrm{mNm}$. The reason why static yield stress is higher than dynamic yield stress is because static yield stress corresponds to an undisturbed, well-connected microstructure while dynamic yield stress corresponds to a broken down microstructure. Thus, thixotropy is assumed to play a big role in the discrepancy between static and dynamic yield stress. It is noted that through a simple thixotropy model, Coussot et al. successfully modeled both the negative slope of equilibrium flow curves and viscosity bifurcation [56]. These two phenomena are associated with dynamic and static yield stress measurements, respectively, thus further supporting that yielding behavior is closely linked to thixotropy.

To investigate the relationship between thixotropy and yield stress, we apply a protocol where the flocculation state of mortar is brought to an equilibrium state under constant angular velocities then subjected to creep, as shown in Fig. 14(a). The preshears are at high and low applied angular velocities, 30 and $1 \mathrm{rad} / \mathrm{s}$, which lead to different flocculation states. And the creep torques are above and below the static or dynamic yield stress.

Fig. 14(b) shows the results of creep when the material is initially brought to equilibrium at $30 \mathrm{rad} / \mathrm{s}$, which is higher than the critical angular velocity ( $7 \mathrm{rad} / \mathrm{s}$ in this study) in the equilibrium flow curve. The applied creep torques are higher and lower than the dynamic yield stress. Recalling the dynamic yield stress was found to be $41.7 \mathrm{mNm}$ based on the Bingham model, applied torques were set to 50 and $40 \mathrm{mNm}$, respectively. When creep is applied at 50 $\mathrm{mNm}$, the mortar continues flowing and stabilizes at $27.3 \mathrm{rad} / \mathrm{s}$. In 

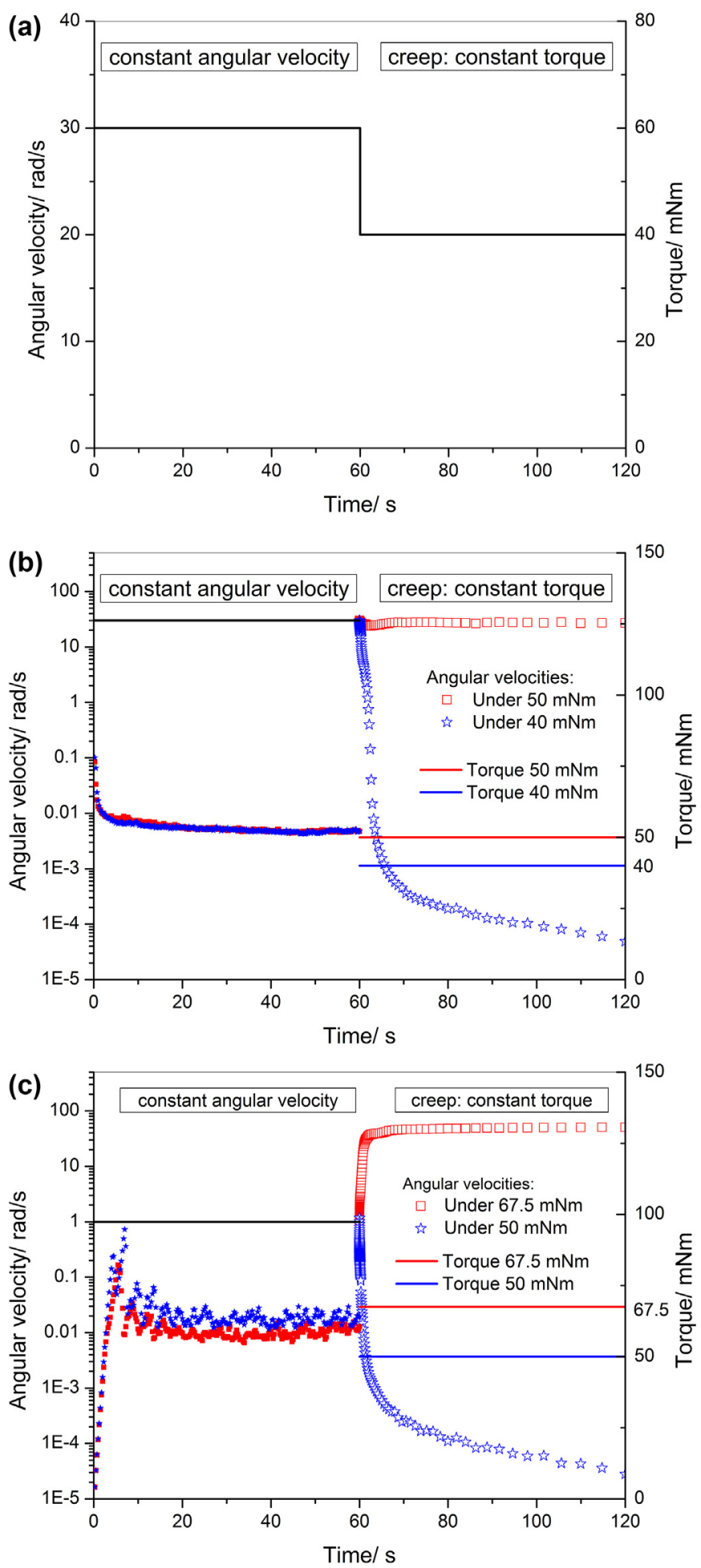

Fig. 14. (a) Preshear-creep protocol; (b) creep after shear at $30 \mathrm{rad} / \mathrm{s}$ for $60 \mathrm{~s}$ and (c) creep after shear at $1 \mathrm{rad} / \mathrm{s}$ for $60 \mathrm{~s}$.

contrast, at $40 \mathrm{mNm}$ the angular velocity decreases to zero.

The creep behavior of mortar when it is brought to equilibrium under $1 \mathrm{rad} / \mathrm{s}$, a lower angular velocity than the critical value in the equilibrium flow curve, is shown in Fig. 14(c). The applied torques for creep are below and above the static yield stress, 50 and 67.5 $\mathrm{mNm}$, respectively. At $50 \mathrm{mNm}$ the angular velocity decreases to zero, indicating the material stops flowing. In the previous case, as shown in Fig. 14(b), where the material is strongly presheared at $30 \mathrm{rad} / \mathrm{s}, 50 \mathrm{mNm}$ is strong enough to break down the structure and keep flowing. However, in the case where the material is not strongly presheared, $50 \mathrm{mNm}$ is not sufficient to break the microstructure of the material. It should be noted that $50 \mathrm{mNm}$ is above the dynamic yield stress and below the static yield stress. When creep is applied at $67.5 \mathrm{mNm}$, which is higher than the static yield stress, the mortar continues to flow and the angular velocity increases to approximately $50 \mathrm{rad} / \mathrm{s}$.

\section{Conclusions}

Dynamic yield stress and static yield stress of mortars are measured through constant applied angular velocity and creep recovery, respectively. A negative slope is captured in the steadystate equilibrium flow curve. Two possible mechanisms, shear localization and sand shear migration, are discussed to explain the negative slope. The flow behavior under constant angular velocity varies depending on angular velocity: at low angular velocities, shear banding and stick slip occur; at medium angular velocities, shear localization occurs and the equilibrium torque decreases with angular velocity; at high angular velocities, the equilibrium torque increases with angular velocity. Dynamic yield stress is obtained through fitting the flow curve at high angular velocities with the Bingham model.

Bifurcation behavior is observed during the creep recovery test. Under low torque, the angular deformation reaches equilibrium after sufficient creep time, showing the flow stops eventually. The equilibrium angular deformation increases with increasing creep torque until a critical point is reached, beyond which only a slight increase in torque causes the angular deformation to increase dramatically and continue to increase, showing the flow continues. In terms of angular velocity, it either decreases to null or increases to an elevated value, indicating flow stops or continues, respectively. This bifurcation behavior is tied to static yield stress.

Finally, using a preshear-creep protocol, it is observed that under different preshear conditions the mortar exhibits different creep behavior and the discrepancy in static and dynamic yield stress is related to thixotropy. The apparent dependence of creep behavior on preshear condition underscores the importance of considering thixotropy and the flocculation state in characterizing viscoelastic properties.

\section{Acknowledgements}

The authors would like to acknowledge the National Science Foundation (NSF EEC-1342377) and Columbia University's Office of the Provost's Grants Program for financial support, and technical support by the staff of Columbia University's Carleton Laboratory.

\section{References}

[1] H. Barnes, K. Walters, The yield stress myth? Rheol. Acta 24 (1985) 323-326.

[2] H.A. Barnes, The'Yield stress Myth?'Paper-21 Years on, Appl. Rheol. 17 (2007), 43110-44250.

[3] H.A. Barnes, The yield stress-a review or ' $\pi \alpha \nu \tau \alpha$ $\rho \varepsilon$ '-everything flows? J. Newt. Fluid Mech. 81 (1999) 133-178.

[4] J.P. Hartnett, R.Y. Hu, Technical note: the yield stress-an engineering reality, J. Rheology (1978-present) 33 (1989) 671-679.

[5] P. Moller, A. Fall, V. Chikkadi, D. Derks, D. Bonn, An attempt to categorize yield stress fluid behaviour, Philosophical Transactions of the Royal Society of London A: Mathematical, Phys. Eng. Sci. 367 (2009) 5139-5155.

[6] C.F. Ferraris, Measurement of the rheological properties of high performance concrete: state of the art report, J. Research-National Inst. Stand. Technol. 104 (1999) 461-478.

[7] N. Roussel, P. Coussot, "Fifty-cent rheometer" for yield stress measurements: from slump to spreading flow, J. Rheology (1978-present) 49 (2005) 705-718.

[8] N. Pashias, D. Boger, J. Summers, D. Glenister, A fifty cent rheometer for yield stress measurement, J. Rheology (1978-present) 40 (1996) 1179-1189. 
[9] A. Pierre, C. Lanos, P. Estellé, Extension of spread-slump formulae for yield stress evaluation, Appl. Rheol. 23 (2013) 63849.

[10] V. Mechtcherine, A. Gram, K. Krenzer, J.-H. Schwabe, C. Bellmann, S. Shyshko, Simulation of fresh concrete flow using Discrete Element Method (DEM), in: Simulation of Fresh Concrete Flow, Springer, 2014, pp. 65-98.

[11] E.C. Bingham, Fluidity and plasticity, McGraw-Hill Book Compny, Incorporated, 1922.

[12] J. Assaad, K.H. Khayat, Assessment of thixotropy of self-consolidating concrete and concrete-equivalent-mortar- effect of binder composition and content, ACI Mater. J. 101 (2004).

[13] J. Assaad, K.H. Khayat, H. Mesbah, Assessment of thixotropy of flowable and self-consolidating concrete, ACI Mater. J. 100 (2003).

[14] J.J. Assaad, K.H. Khayat, Effect of viscosity-enhancing admixtures on formwork pressure and thixotropy of self-consolidating concrete, ACI Mater. J. 103 (2006).

[15] L.J. Struble, M.A. Schultz, Using creep and recovery to study flow behavior of fresh cement paste, Cem. Concr. Res. 23 (1993) 1369-1379.

[16] F. De Larrard, C. Ferraris, T. Sedran, Fresh concrete: a Herschel-Bulkley material, Mater Struct. 31 (1998) 494-498.

[17] A. Yahia, K. Khayat, Analytical models for estimating yield stress of highperformance pseudoplastic grout, Cem. Concr. Res. 31 (2001) 731-738.

[18] N. Roussel, G. Ovarlez, S. Garrault, C. Brumaud, The origins of thixotropy of fresh cement pastes, Cem. Concr. Res. 42 (2012) 148-157.

[19] H.A. Barnes, Thixotropy - a review, J. Newt. Fluid Mech. 70 (1997) 1-33.

[20] R. Lapasin, V. Longo, S. Rajgelj, Thixotropic behaviour of cement pastes, Cem. Concr. Res. 9 (1979) 309-318.

[21] J.E. Wallevik, Rheological properties of cement paste: thixotropic behavior and structural breakdown, Cem. Concr. Res. 39 (2009) 14-29.

[22] J.E. Wallevik, Thixotropic investigation on cement paste: experimental and numerical approach, J. Newt. fluid Mech. 132 (2005) 86-99.

[23] D.C. Cheng, Yield stress: a time-dependent property and how to measure it, Rheol. Acta 25 (1986) 542-554.

[24] P.C. Møller, J. Mewis, D. Bonn, Yield stress and thixotropy: on the difficulty of measuring yield stresses in practice, Soft matter 2 (2006) 274-283.

[25] F. Mahaut, S. Mokeddem, X. Chateau, N. Roussel, G. Ovarlez, Effect of coarse particle volume fraction on the yield stress and thixotropy of cementitious materials, Cem. Concr. Res. 38 (2008) 1276-1285.

[26] Y. Qian, S. Kawashima, Use of creep recovery protocol to measure static yield stress and structural rebuilding of fresh cement pastes, Cem. Concr. Res. 90 (2016) 73-79.

[27] D. Cheng, Measurement Techniques for Thixotropic Properties, Paper to Joint British, Italian and Netherlands, Society of Rheology, British Society of Rheology, Amsterdam, 1979.

[28] D.-H. Cheng, R. Richmond, Determination of the thixotropic property of an emulsion gel paint, in: Rheology, Springer, 1980, pp. 575-580.

[29] T. Davenport, R. Somper, The yield value and breakdown of crude oil gels, J. Inst. Pet. 57 (1971) 86-105.

[30] E.P. Koehler, D.W. Fowler, Summary of concrete workability test methods, ICAR Tech. Rep. (2003).

[31] J.E. Wallevik, Relationship between the Bingham parameters and slump, Cem. Concr. Res. 36 (2006) 1214-1221.

[32] J. Assaad, K.H. Khayat, Variations of lateral and pore water pressure of selfconsolidating concrete at early age, ACI Mater. J. 101 (2004).

[33] K. Khayat, J. Assaad, H. Mesbah, M. Lessard, Effect of section width and casting rate on variations of formwork pressure of self-consolidating concrete, Mater Struct. 38 (2005) 73-78.

[34] G. Ovarlez, N. Roussel, A physical model for the prediction of lateral stress exerted by self-compacting concrete on formwork, Mater Struct. 39 (2006)
$269-279$.

[35] J. Tchamba, S. Amziane, G. Ovarlez, N. Roussel, Lateral stress exerted by fresh cement paste on formwork: laboratory experiments, Cem. Concr. Res. 38 (2008) 459-466.

[36] S. Kawashima, J.H. Kim, D.J. Corr, S.P. Shah, Study of the mechanisms underlying the fresh-state response of cementitious materials modified with nanoclays, Constr. Build. Mater. 36 (2012) 749-757.

[37] P. Billberg, Form pressure generated by self-compacting concrete: influence of thixotropy and structural behaviour at rest, 2006.

[38] N. Roussel, A theoretical frame to study stability of fresh concrete, Mater Struct. 39 (2006) 81-91.

[39] N. Roussel, F. Cussigh, Distinct-layer casting of SCC: the mechanical consequences of thixotropy, Cem. Concr. Res. 38 (2008) 624-632.

[40] T. Wangler, E. Lloret, L. Reiter, N. Hack, F. Gramazio, M. Kohler, M. Bernhard B. Dillenburger, J. Buchli, N. Roussel, Digital concrete: opportunities and challenges, RILEM Tech. Lett. 1 (2016) 67-75.

[41] Y. Qian, S. Kawashima, Flow onset of fresh mortars in rheometers: contribution of paste deflocculation and sand particle migration, Cem. Concr. Res. 90 (2016) 97-103.

[42] M. Reiner, Deformation and flow: an elementary introduction to theoretical rheology, HK Lewis (1949).

[43] D. Feys, J.E. Wallevik, A. Yahia, K.H. Khayat, O.H. Wallevik, Extension of the Reiner-Riwlin equation to determine modified Bingham parameters measured in coaxial cylinders rheometers, Mater Struct. 46 (2013) 289-311.

[44] P. Estellé, C. Lanos, A. Perrot, Processing the Couette viscometry data using a Bingham approximation in shear rate calculation, J. Newt. Fluid Mech. 154 (2008) 31-38.

[45] G. Ovarlez, F. Mahaut, S. Deboeuf, N. Lenoir, S. Hormozi, X. Chateau, Flows of suspensions of particles in yield stress fluids, J. Rheology (1978-present) 59 (2015) 1449-1486.

[46] H. Hafid, G. Ovarlez, F. Toussaint, P. Jezequel, N. Roussel, Assessment of potential concrete and mortar rheometry artifacts using magnetic resonance imaging, Cem. Concr. Res. 71 (2015) 29-35.

[47] O.H. Wallevik, D. Feys, J.E. Wallevik, K.H. Khayat, Avoiding inaccurate interpretations of rheological measurements for cement-based materials, Cem. Concr. Res. (2015).

[48] J. Spangenberg, N. Roussel, J. Hattel, H. Stang, J. Skocek, M. Geiker, Flow induced particle migration in fresh concrete: theoretical frame, numerica simulations and experimental results on model fluids, Cem. Concr. Res. 42 (2012) 633-641.

[49] J.J. Assaad, Correlating water extraction to viscosity variations of injection grouts, Constr. Build. Mater. 77 (2015) 74-82.

[50] P. Møller, S. Rodts, M. Michels, D. Bonn, Shear banding and yield stress in soft glassy materials, Phys. Rev. E 77 (2008) 041507.

[51] N. Huang, G. Ovarlez, F. Bertrand, S. Rodts, P. Coussot, D. Bonn, Flow of wet granular materials, Phys. Rev. Lett. 94 (2005) 028301.

[52] S. Jarny, N. Roussel, S. Rodts, F. Bertrand, R. Le Roy, P. Coussot, Rheological behavior of cement pastes from MRI velocimetry, Cem. Concr. Res. 35 (2005) 1873-1881.

[53] P. Coussot, L. Tocquer, C. Lanos, G. Ovarlez, Macroscopic vs. local rheology of yield stress fluids, J. Newt. Fluid Mech. 158 (2009) 85-90.

[54] P. Coussot, Q.D. Nguyen, H. Huynh, D. Bonn, Avalanche behavior in yield stress fluids, Phys. Rev. Lett. 88 (2002) 175501.

[55] J.J. Assaad, J. Harb, Y. Maalouf, Effect of vane configuration on yield stress measurements of cement pastes, J. Newt. Fluid Mech. 230 (2016) 31-42.

[56] P. Coussot, Q. Nguyen, H. Huynh, D. Bonn, Viscosity bifurcation in thixotropic, yielding fluids, J. Rheology (1978-present) 46 (2002) 573-589. 